\title{
A consensus reaching process in the context of non-uniform ordered qualitative scales
}

\author{
José Luis García-Lapresta \\ PRESAD Research Group, BORDA Research Unit, IMUVA, Dept. de Economía Aplicada, \\ Universidad de Valladolid, Spain \\ David Pérez-Román \\ PRESAD Research Group, BORDA Research Unit, Dept. de Organización de Empresas y \\ Comercialización e Investigación de Mercados, Universidad de Valladolid, Spain
}

\begin{abstract}
In this paper, we consider that a group of agents judge a set of alternatives by means of an ordered qualitative scale. The scale is not assumed to be uniform, i.e., the psychological distance between adjacent linguistic terms is not necessarily always the same. In this setting, we propose how to measure the consensus in each subset of at least two agents over each subset of alternatives. We introduce a consensus reaching process where some agents may be invited to change their assessments over some alternatives in order to increase the consensus. All the steps are managed in a purely ordinal way through ordinal proximity measures.

Keywords: group decision making; consensus; qualitative scales; ordinal proximity measures.
\end{abstract}

\section{Introduction}

In some group decision making problems, agents show their opinions on a set of alternatives and a voting system generates an outcome (a winning alternative, a set of winning alternatives, a ranking on the set of alternatives, etc.). When the agreement among the agents is low, the outcome might not faithfully represent the opinions of a large number of agents, and they could feel that the outcome is not acceptable. In these cases, the outcome might be very different across voting systems ${ }^{1}$. For these reasons, it is important to reach as highest consensus as possible before applying a voting system.

Email addresses: lapresta@eco.uva.es (José Luis García-Lapresta), david@emp.uva.es (David Pérez-Román)

${ }^{1}$ For instance, Malkevitch [20] provides an "evil example" where six well-known voting systems generate different outcomes from the same profile of individual preferences.

Preprint submitted to Fuzzy Optimization and Decision Making 


\subsection{Consensus measures}

For measuring the degree of consensus among a group of agents that provide their opinions on a set of alternatives, different proposals can be found in the literature.

In the fuzzy framework, there exists a huge literature where different consensus measures have been proposed (see Pérez et al. [22] and Cabrerizo et al. [7, 6], among others). A referenced survey can be found in Palomares et al. [21].

More recently, the Social Choice Theory has been interested on how to measure consensus in different settings. First, the notion of consensus measure was introduced by Bosch [4] in the context of linear orders. García-Lapresta and Pérez-Román [13] extended that notion to the context of weak orders and they analyzed a class of consensus measures generated by distances. In turn, Erdamar et al. [10] extended the notion of consensus measure to the preference-approval setting through different kinds of distances. García-Lapresta and Pérez-Román [16] provides a distance-based family of consensus measures generated by aggregation functions in the context of hesitant qualitative assessments.

\subsection{Consensus reaching processes}

All the above mentioned consensus analyses are static: once the agents show their opinions, a degree of consensus is generated by a specific consensus measure. In consensus reaching processes, the degree of consensus in a specific situation is only the starting point of a dynamic and iterative procedure that pursues to increase the agreement among agents.

A consensus reaching process consists of several rounds where some agents may be invited to modify their opinions in order to increase the collective agreement. These rounds can be conducted by a human or virtual moderator (see Fedrizzi et al. [11], Saint and Lawson [23], Herrera et al. [18], Herrera-Viedma et al. [19], Cabrerizo et al. [6] and Wu and Chiclana [25], among others). Once a previously fixed consensus threshold is reached, a group decision making procedure can be carried out in order to rank the alternatives or to select a winning alternative.

\subsection{Ordinal proximity measures}

The notion of ordinal proximity was introduced by García-Lapresta and Pérez-Román [14] for measuring, in a purely ordinal way, the psychological proximities between linguistic terms of ordered qualitative scales. That approach has some similarities with difference measurement within the classical measurement theory, and also with non-metric multidimensional scaling, where only the ranks of the psychological distances or proximities are known.

\subsection{Our proposal}

In this paper, we consider that agents evaluate the alternatives through an ordered qualitative scale (non necessarily uniform, in the sense that the psychological distance between adjacent linguistic terms is not necessarily always the same). We introduce a degree of consensus in each subset of at least two agents 
over each subset of alternatives as the outcome generated by a median operator to the ordinal proximities between the pairs of individual assessments.

Given a consensus threshold, if the overall degree of consensus reaches that threshold, then a voting system is applied. Otherwise, a consensus reaching process starts. In this case, first we select the alternatives where the consensus among the agents is smaller than the overall degree of consensus. For each of these alternatives, we select the agents whose assessments are either sufficiently higher or lower than the median assessment according to a previously fixed proximity threshold. These agents are invited to change their assessments in order to increase the overall degree of consensus. This process is iterated until the overall degree of consensus reaches the consensus threshold.

The rest of the paper is organized as follows. In Section 2 we introduce basic notation and ordinal proximity measures. Section 3 is devoted to consensus measures and the consensus reaching process. Finally, Section 4 includes some conclusions.

\section{Preliminaries}

We consider that each individual of a group of agents assesses every alternative with a linguistic term of an ordered qualitative scale $\mathcal{L}=\left\{l_{1}, \ldots, l_{g}\right\}$, arranged from the lowest to the highest terms, where the granularity of $\mathcal{L}$ is at least 3, i.e., $g \geq 3$.

For instance, Balinski and Laraki [1] use the following six linguistic terms for assessing candidates in political elections: 'to reject' $\left(l_{1}\right)$, 'poor' $\left(l_{2}\right)$, 'acceptable' $\left(l_{3}\right)$, 'good' $\left(l_{4}\right)$, 'very good' $\left(l_{5}\right)$ and 'excellent' $\left(l_{6}\right)$. In turn, Balinski and Laraki [2] consider the following seven linguistic terms used by the International Association of Oenologists for assessing wines and spirits ${ }^{2}$ : 'bad' $\left(l_{1}\right)$, 'mediocre' $\left(l_{2}\right)$, 'inadequate' $\left(l_{3}\right)$, 'passable' $\left(l_{4}\right)$, 'good' $\left(l_{5}\right)$, 'very good' $\left(l_{6}\right)$ and 'excellent' $\left(l_{7}\right)$.

We now recall the notion of ordinal proximity between linguistic terms with values on a finite chain (linear order) $\Delta=\left\{\delta_{1}, \ldots, \delta_{h}\right\}$, with $\delta_{1} \succ \cdots \succ \delta_{h}$, introduced by García-Lapresta and Pérez-Román [14]. The elements of $\Delta$ have no meaning and they only represent different degrees of proximity, being $\delta_{1}$ and $\delta_{h}$ the maximum and minimum degrees, respectively.

As usual in the setting of linear orders, $\delta_{r} \prec \delta_{s}$ means $\delta_{s} \succ \delta_{r} ; \delta_{r} \preceq \delta_{s}$ means $\delta_{r} \prec \delta_{s}$ or $\delta_{r}=\delta_{s}$; and $\delta_{r} \succeq \delta_{s}$ means $\delta_{r} \succ \delta_{s}$ or $\delta_{r}=\delta_{s}$.

First, we assume that all the elements of $\Delta$ are relevant because they are reached as the degree of proximity between at least a pair of linguistic terms (exhaustiveness). We also assume that the proximity between a pair of linguistic terms does not depend on the order these terms are presented (symmetry), and the maximum proximity between linguistic terms is only reached when comparing a term with itself. Additionally, we assume that, given three different

\footnotetext{
${ }^{2}$ We have to note that the International Association of Oenologists assigns numbers to these linguistic terms and does manage the assessments in a numerical way.
} 
linguistic terms, the degree of proximity between the lowest and the highest terms should be smaller than the degrees of proximity between the lowest and the intermediate terms and also between the intermediate and the highest terms (monotonicity).

Definition 1. ([14]) $A n$ ordinal proximity measure on $\mathcal{L}$ with values in $\Delta$ is a mapping $\pi: \mathcal{L}^{2} \longrightarrow \Delta$, where $\pi\left(l_{r}, l_{s}\right)=\pi_{r s}$ means the degree of proximity between $l_{r}$ and $l_{s}$, satisfying the following conditions:

1. Exhaustiveness: For every $\delta \in \Delta$, there exist $l_{r}, l_{s} \in \mathcal{L}$ such that $\delta=\pi_{r s}$.

2. Symmetry: $\pi_{s r}=\pi_{r s}$, for all $r, s \in\{1, \ldots, g\}$.

3. Maximum proximity: $\pi_{r s}=\delta_{1} \Leftrightarrow r=s$, for all $r, s \in\{1, \ldots, g\}$.

4. Monotonicity: $\pi_{r s} \succ \pi_{r t}$ and $\pi_{s t} \succ \pi_{r t}$, for all $r, s, t \in\{1, \ldots, g\}$ such that $r<s<t$.

As shown in García-Lapresta and Pérez-Román [14], the conditions appearing in Definition 1 are independent (Prop. 1), and the minimum proximity between linguistic terms is only reached when comparing the extreme linguistic terms (Prop. 2): $\pi_{r s}=\delta_{h} \Leftrightarrow(r, s) \in\{(1, g),(g, 1)\}$.

Every ordinal proximity measure can be represented by a $g \times g$ symmetric matrix with coefficients in $\Delta$, where the elements in the main diagonal are $\pi_{r r}=\delta_{1}, r=1, \ldots, g$ and $\pi_{1 g}=\delta_{h}$ :

$$
\left(\begin{array}{ccccc}
\pi_{11} & \cdots & \pi_{1 s} & \cdots & \pi_{1 g} \\
\cdots & \cdots & \cdots & \cdots & \cdots \\
\pi_{r 1} & \cdots & \pi_{r s} & \cdots & \pi_{r g} \\
\cdots & \cdots & \cdots & \cdots & \cdots \\
\pi_{g 1} & \cdots & \pi_{g s} & \cdots & \pi_{g g}
\end{array}\right)
$$

This matrix is called proximity matrix.

Taking into account the conditions of Definition 1 and $\pi_{1 g}=\delta_{h}$, the upper half proximity matrix

$$
\left(\begin{array}{cccccc}
\delta_{1} & \pi_{12} & \pi_{13} & \cdots & \pi_{1(g-1)} & \delta_{h} \\
& \delta_{1} & \pi_{23} & \cdots & \pi_{2(g-1)} & \pi_{2 g} \\
& & & \cdots & \cdots & \cdots \\
& & & & \delta_{1} & \pi_{(g-1) g} \\
& & & & & \delta_{1}
\end{array}\right)
$$

totally describes the ordinal proximity measure.

The cardinality of the chain $\Delta$ of ordinal degrees, $h$, is located between the granularity of $\mathcal{L}, g$, and a polinomial of degree 2 of that granularity (see García-Lapresta and Pérez-Román [14, Prop. 4]):

$$
g \leq h \leq \frac{g \cdot(g-1)}{2}+1
$$


In the following example we show two extreme situations for a qualitative scale of four linguistic terms.

Example 1. Consider $g=4$, where five comparisons are needed (see Fig. 1) and $h \in\{4,5,6,7\}$.

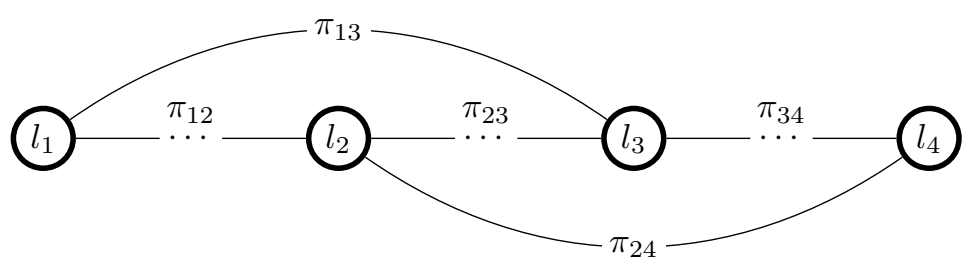

Figure 1: Comparisons for $g=4$.

1. The simplest case corresponds to $\Delta=\left\{\delta_{1}, \delta_{2}, \delta_{3}, \delta_{4}\right\}$, with $\pi_{r r}=\delta_{1}$, $\pi_{12}=\pi_{23}=\pi_{34}=\delta_{2}, \pi_{13}=\pi_{24}=\delta_{3}$ and $\pi_{14}=\delta_{4}$ (the totally uniform scale). It corresponds to the following upper half proximity matrix

$$
\left(\begin{array}{cccc}
\delta_{1} & \delta_{2} & \delta_{3} & \delta_{4} \\
& \delta_{1} & \delta_{2} & \delta_{3} \\
& & \delta_{1} & \delta_{2} \\
& & & \delta_{1}
\end{array}\right)
$$

that can be visualized in Fig. 2 .

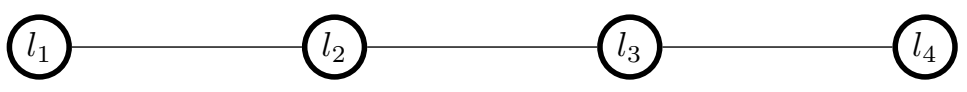

Figure 2: The totally uniform scale for $g=4$.

2. One of the most complex cases corresponds to $\Delta=\left\{\delta_{1}, \delta_{2}, \delta_{3}, \delta_{4}, \delta_{5}, \delta_{6}, \delta_{7}\right\}$, with $\pi_{r r}=\delta_{1}, \pi_{34}=\delta_{2}, \pi_{23}=\delta_{3}, \pi_{12}=\delta_{4}, \pi_{24}=\delta_{5}, \pi_{13}=\delta_{6}$ and $\pi_{14}=\delta_{7}$. It corresponds to the following upper half proximity matrix

$$
\left(\begin{array}{cccc}
\delta_{1} & \delta_{4} & \delta_{6} & \delta_{7} \\
& \delta_{1} & \delta_{3} & \delta_{5} \\
& & \delta_{1} & \delta_{2} \\
& & & \delta_{1}
\end{array}\right)
$$

that can be visualized in Fig. 3 . 


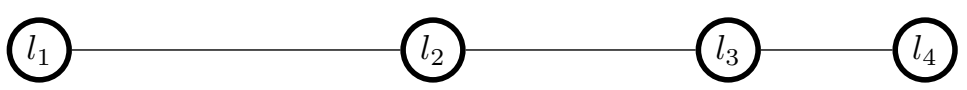

Figure 3: A non-uniform scale for $g=4$.

\section{The consensus reaching process}

Consider a set of agents $A=\{1, \ldots, m\}$, with $m \geq 2$, that have to evaluate a set of alternatives $X=\left\{x_{1}, \ldots, x_{n}\right\}$, with $n \geq 2$, through an ordered qualitative scale $\mathcal{L}=\left\{l_{1}, \ldots, l_{g}\right\}, l_{1}<\cdots<l_{g}$, with $g \geq 3$. We also assume that an ordinal proximity measure on $\mathcal{L}$ with values on $\Delta=\left\{\delta_{1}, \ldots, \delta_{h}\right\}, \pi: \mathcal{L}^{2} \longrightarrow \Delta$, has been fixed.

The judgments of agents on the alternatives are collected in a profile, that is a matrix

$$
V=\left(\begin{array}{ccccc}
v_{1}^{1} & \cdots & v_{i}^{1} & \cdots & v_{n}^{1} \\
\cdots & \cdots & \cdots & \cdots & \cdots \\
v_{1}^{a} & \cdots & v_{i}^{a} & \cdots & v_{n}^{a} \\
\cdots & \cdots & \cdots & \cdots & \cdots \\
v_{1}^{m} & \cdots & v_{i}^{m} & \cdots & v_{n}^{m}
\end{array}\right)=\left(v_{i}^{a}\right)
$$

consisting of $m$ rows and $n$ columns of linguistic terms, where the element $v_{i}^{a} \in \mathcal{L}$ represents the linguistic assessment given by the agent $a \in A$ to the alternative $x_{i} \in X$.

\subsection{Ranking medians of ordinal degrees}

In the Majority Judgment voting system, Balinski and Laraki [1] assign the median (lower median) of the linguistic individual assessments as collective degree of each alternative whenever the number of assessments is odd (even $)^{3}$. In the even case, that assignment is not problematic when the number of assessments is high, as happens in political elections. However, it can be considered arbitrary when that number is low, for instance in small committees.

In our setting, for each subset of alternatives and each subset of at least two agents, we shall consider the degrees of ordinal proximity among the linguistic assessments obtained by these alternatives. Next we select the median(s) of the mentioned ordinal degrees for defining a consensus measure ${ }^{4}$.

Given a vector of ordinal degrees $\delta=\left(\delta_{1}, \ldots, \delta_{p}\right) \in \Delta^{p}$, we arrange its components in a decreasing fashion, from the highest to the lowest degrees. If $p$ is odd, then the median of $\boldsymbol{\delta}$ is unique, say $\delta_{r} \in \Delta$. However, if $p$ is even, then $\delta$ has two medians, say $\delta_{s}, \delta_{t} \in \Delta$ such that $s \leq t$, i.e., $\delta_{s} \succeq \delta_{t}$. In order to unify the assignment of medians, we consider the pair of medians $\left(\delta_{r}, \delta_{r}\right)$ and $\left(\delta_{s}, \delta_{t}\right)$ whenever $p$ is odd and even, respectively.

\footnotetext{
${ }^{3}$ In Majority Judgment the inputs are arranged in an increasing fashion.

${ }^{4}$ In order to avoid loss of information, we will take into account the two medians in the even case.
} 
More formally, given the set of feasible medians $\Delta_{2}=\left\{\left(\delta_{r}, \delta_{s}\right) \in \Delta^{2} \mid r \leq s\right\}$, the median operator is the mapping

$$
M: \bigcup_{p=1}^{\infty} \Delta^{p} \longrightarrow \Delta_{2}
$$

that assigns the corresponding pair of medians to each vector of ordinal degrees.

For ranking the medians of ordinal proximities among the linguistic assessments obtained by different alternatives, we propose an appropriate linear order on the set of feasible medians ${ }^{5}$.

Let $\succeq$ be the linear order on $\Delta_{2}$ defined as

$$
\left(\delta_{r}, \delta_{s}\right) \succeq\left(\delta_{t}, \delta_{u}\right) \Leftrightarrow\left\{\begin{array}{l}
r+s<t+u \\
\text { or } \\
r+s=t+u \text { and } s-r \leq u-t,
\end{array}\right.
$$

for all $\left(\delta_{r}, \delta_{s}\right),\left(\delta_{t}, \delta_{u}\right) \in \Delta_{2}$.

It is easy to see that if $r+s=t+u$, then $s-r \leq u-t \Leftrightarrow s \leq u$. Thus, (1) can be rewritten as follows:

$$
\left(\delta_{r}, \delta_{s}\right) \succeq\left(\delta_{t}, \delta_{u}\right) \Leftrightarrow\left\{\begin{array}{l}
r+s<t+u \\
\text { or } \\
r+s=t+u \text { and } s \leq u,
\end{array}\right.
$$

for all $\left(\delta_{r}, \delta_{s}\right),\left(\delta_{t}, \delta_{u}\right) \in \Delta_{2}$.

For example, if $\Delta=\left\{\delta_{1}, \delta_{2}, \delta_{3}, \delta_{4}, \delta_{5}, \delta_{6}\right\}$, the linear order $\succeq$ produces the following ranking in $\Delta_{2}$ :

$$
\begin{aligned}
& \left(\delta_{1}, \delta_{1}\right) \succ\left(\delta_{1}, \delta_{2}\right) \succ\left(\delta_{2}, \delta_{2}\right) \succ\left(\delta_{1}, \delta_{3}\right) \succ\left(\delta_{2}, \delta_{3}\right) \succ\left(\delta_{1}, \delta_{4}\right) \succ\left(\delta_{3}, \delta_{3}\right) \succ \\
& \left(\delta_{2}, \delta_{4}\right) \succ\left(\delta_{1}, \delta_{5}\right) \succ\left(\delta_{3}, \delta_{4}\right) \succ\left(\delta_{2}, \delta_{5}\right) \succ\left(\delta_{1}, \delta_{6}\right) \succ\left(\delta_{4}, \delta_{4}\right) \succ\left(\delta_{3}, \delta_{5}\right) \succ \\
& \left(\delta_{2}, \delta_{6}\right) \succ\left(\delta_{4}, \delta_{5}\right) \succ\left(\delta_{3}, \delta_{6}\right) \succ\left(\delta_{5}, \delta_{5}\right) \succ\left(\delta_{4}, \delta_{6}\right) \succ\left(\delta_{5}, \delta_{6}\right) \succ\left(\delta_{6}, \delta_{6}\right) .
\end{aligned}
$$

Obviously, other linear orders on $\Delta_{2}$ might be considered.

\subsection{The sequential process}

We first introduce a consensus measure that is related to the one introduced by García-Lapresta and Pérez-Román [14].

Given a set $I$, with $\# I$ we denote the cardinality of $I$. With $\mathcal{P}(X)$ we denote the power set of $X$, and $\mathcal{P}_{2}(A)=\{I \subseteq A \mid \# I \geq 2\}$ denotes the family of subsets of $A$ with at least two agents.

\footnotetext{
${ }^{5}$ This linear order is equivalent to the one provided by Delgado et al. [8] in the set of trapezoidal fuzzy numbers when it is applied to intervals of real numbers.
} 
Definition 2. Given a profile $V=\left(v_{i}^{a}\right)$, the associated consensus measure $C$ is the mapping

$$
C: \mathcal{P}_{2}(A) \times(\mathcal{P}(X) \backslash\{\emptyset\}) \longrightarrow \Delta_{2}
$$

defined as

$$
C(I, Y)=M\left(\pi\left(v_{i}^{a}, v_{i}^{b}\right)_{\substack{a, b \in I, a<b \\ x_{i} \in Y}}\right) .
$$

The overall degree of consensus is defined as $C(A, X)$ and it measures the agreement of all the agents about all the alternatives.

We now introduce the consensus reaching process. It is related to the one introduced by García-Lapresta et al. [17] (in a different setting).

Given a profile $V=\left(v_{i}^{a}\right)$, with $\boldsymbol{v}_{i}$ we denote the assessments vector of alternative $x_{i}$, i.e., $\boldsymbol{v}_{i}=\left(v_{i}^{1}, \ldots, v_{i}^{m}\right) \in \mathcal{L}^{m}$. With $v_{i}^{+}$and $v_{i}^{-}$we denote the upper median and the lower median, respectively, of the data distribution given by $\boldsymbol{v}_{i}$ when they are arranged in an increasing fashion. Notice that if $m$ is odd, then the median is unique and, consequently, $v_{i}^{+}=v_{i}^{-}$.

Before starting to ask the agents their opinions on the alternatives, a consensus threshold $\gamma \in \Delta$ is fixed.

1. If $C(A, X) \succeq(\gamma, \gamma)$, then a voting system is applied ${ }^{6}$.

2. If $C(A, X) \prec(\gamma, \gamma)$, then the moderator initiates a consensus reaching process. In that case, the set of alternatives where the degrees of consensus are lower than the overall degree of consensus is determined:

$$
X^{-}=\left\{x_{i} \in X \mid C\left(A,\left\{x_{i}\right\}\right) \prec C(A, X)\right\} .
$$

For each $x_{i} \in X^{-}$, we define

$$
A_{i}^{+}=\left\{a \in A \mid v_{i}^{a}>v_{i}^{+}\right\} \text {and } A_{i}^{-}=\left\{a \in A \mid v_{i}^{a}<v_{i}^{-}\right\} .
$$

Given a proximity threshold $\delta \in \Delta$,

1. If $a \in A_{i}^{+}$and $\pi\left(v_{i}^{a}, v_{i}^{+}\right) \prec \delta$, then agent $a$ is invited to decrease $v_{i}^{a}$ with $v_{i}^{-}$as lowest assessment (see Fig. 4).

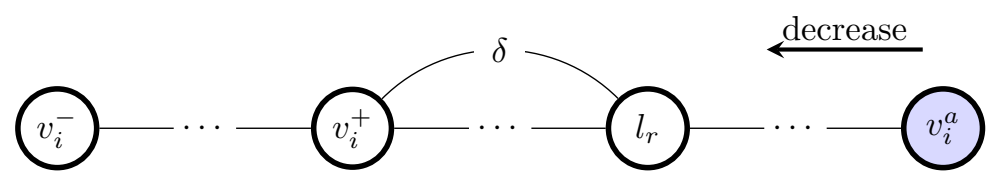

Figure 4: Recommendation to $a \in A_{i}^{+}$.

\footnotetext{
${ }^{6}$ For instance, the one introduced by García-Lapresta and Pérez-Román [15] in the same setting.
} 
2. If $a \in A_{i}^{-}$and $\pi\left(v_{i}^{a}, v_{i}^{-}\right) \prec \delta$, then agent $a$ is invited to increase $v_{i}^{a}$ with $v_{i}^{+}$as highest assessment (see Fig. 5).

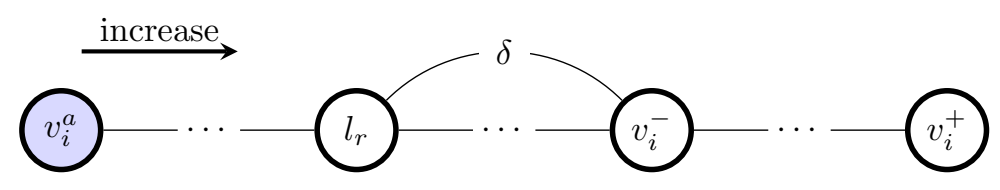

Figure 5: Recommendation to $a \in A_{i}^{-}$.

Once the new profile is obtained, the degrees of consensus are calculated and the process is re-initiated (see Fig. 6).

Remark 1. The following remarks are relevant for the consensus reaching process.

1. The number of rounds has to be fixed previously to the consensus reaching process. If the consensus threshold $\gamma$ is reached before finishing the maximum number of rounds, then the voting system is applied. But if the rounds finish without reaching the consensus threshold, then it would be necessary to adopt a strategy (see Saint and Lawson [23]). One possibility is to exclude the agents whose overall contributions to consensus are negative, as in García-Lapresta [12].

2. The proximity threshold $\delta \in \Delta$ may be modified for facilitating the achievement of an increase of the overall degree of consensus. If the threshold is high, then the number of recommendations can be very high. On the contrary, if the threshold is low, then the number of recommendations can be insufficient for achieving an increase of the overall degree of consensus.

3. The consensus and proximity thresholds might be chosen in the richer set $\Delta_{2}$ instead of $\Delta:\left(\gamma_{1}, \gamma_{2}\right) \in \Delta_{2}$ and $\left(\delta_{1}, \delta_{2}\right) \in \Delta_{2}$, respectively. This increases the leeway from $\# \Delta=h$ to $\# \Delta_{2}=\frac{(h+1) \cdot h}{2}$.

\subsection{An illustrative example}

In order to illustrate how the consensus reaching process works, we now show an illustrative example. Consider a firm has to choose a building for establishing the headquarters. After a previous analysis of different possibilities, taking into account price, size dimensions, location, functionality and other attributes, the firm has selected three admissible buildings. In order to make the final decision, five experts have to judge these three alternatives according to criterion location by means of a non-uniform qualitative scale composed by four linguistic terms.. The set of agents is $A=\{1,2,3,4,5\}$, the set of alternatives is $X=\left\{x_{1}, x_{2}, x_{3}\right\}$, and the qualitative scale is $\mathcal{L}=\left\{l_{1}, l_{2}, l_{3}, l_{4}\right\}$ with the meanings appearing in Table 1. 


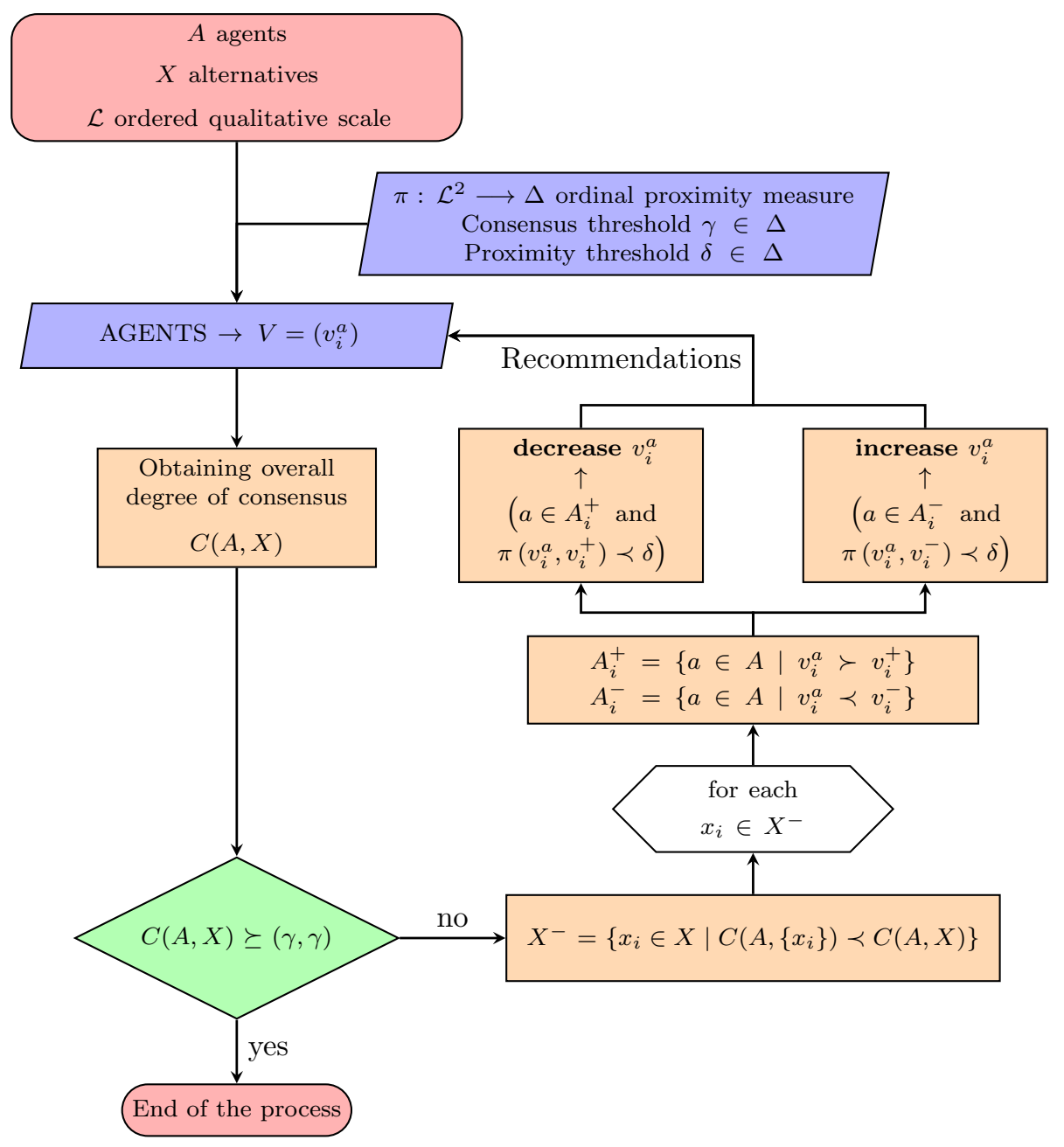

Figure 6: Flowchart.

Before starting to ask the agents their opinions on the alternatives, a consensus threshold $\gamma=\delta_{3} \in \Delta$ and a proximity threshold $\delta=\delta_{3} \in \Delta$ are fixed.

The assessments of the agents on the alternatives are collected in the following profile

$$
V=\left(\begin{array}{ccc}
v_{1}^{1} & v_{2}^{1} & v_{3}^{1} \\
v_{1}^{2} & v_{2}^{2} & v_{3}^{2} \\
v_{1}^{3} & v_{2}^{3} & v_{3}^{3} \\
v_{1}^{4} & v_{2}^{4} & v_{3}^{4} \\
v_{1}^{5} & v_{2}^{5} & v_{3}^{5}
\end{array}\right)=\left(\begin{array}{ccc}
l_{2} & l_{1} & l_{1} \\
l_{2} & l_{1} & l_{2} \\
l_{2} & l_{2} & l_{2} \\
l_{3} & l_{2} & l_{3} \\
l_{4} & l_{3} & l_{4}
\end{array}\right) .
$$

1. Consider the first case of Example 1 (the totally uniform scale). It is easy 


\begin{tabular}{cccc}
$l_{1}$ & $l_{2}$ & $l_{3}$ & $l_{4}$ \\
\hline bad & acceptable & good & very good
\end{tabular}

Table 1: Meaning of the linguistic terms.

to see that $C(A, X)=\left(\delta_{2}, \delta_{2}\right) \succeq\left(\delta_{3}, \delta_{3}\right)$; then, a voting system is applied. 2. We now take into account $\Delta=\left\{\delta_{1}, \delta_{2}, \delta_{3}, \delta_{4}, \delta_{5}, \delta_{6}, \delta_{7}\right\}$, with $\pi_{r r}=\delta_{1}$, $\pi_{12}=\delta_{5}, \pi_{13}=\delta_{6}, \pi_{14}=\delta_{7}, \pi_{23}=\delta_{3}, \pi_{24}=\delta_{4}, \pi_{34}=\delta_{2}$ and $\pi_{14}=\delta_{7}$. It corresponds to the following upper half proximity matrix

$$
\left(\begin{array}{cccc}
\delta_{1} & \delta_{5} & \delta_{6} & \delta_{7} \\
& \delta_{1} & \delta_{3} & \delta_{4} \\
& & \delta_{1} & \delta_{2} \\
& & & \delta_{1}
\end{array}\right)
$$

that can be visualized in Fig. 7 .

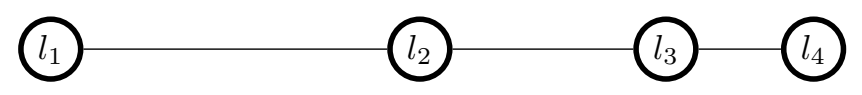

Figure 7: The non-uniform scale.

In this case, $C(A, X)=\left(\delta_{3}, \delta_{4}\right) \prec\left(\delta_{3}, \delta_{3}\right)$. The set of alternatives where the degrees of consensus are lower than the overall degree of consensus is $X^{-}=\left\{x_{2}, x_{3}\right\}$, because $C\left(A,\left\{x_{2}\right\}\right)=\left(\delta_{5}, \delta_{5}\right) \prec\left(\delta_{3}, \delta_{4}\right)=C(A, X)$ and $C\left(A,\left\{x_{3}\right\}\right)=\left(\delta_{4}, \delta_{4}\right) \prec\left(\delta_{3}, \delta_{4}\right)=C(A, X)$.

Taking into account that $v_{2}^{-}=v_{2}^{+}=v_{3}^{-}=v_{3}^{+}=l_{2}$, we have $A_{2}^{+}=\{5\}$, because $v_{2}^{5}=l_{3}>l_{2} ; A_{2}^{-}=\{1,2\}$, because $v_{2}^{1}=v_{2}^{2}=l_{1}<l_{2} ; A_{3}^{+}=$ $\{4,5\}$, because $v_{3}^{4}=l_{3}>l_{2}$ and $v_{3}^{5}=l_{4}>l_{2}$; and $A_{3}^{-}=\{1\}$, because $v_{3}^{1}=l_{1}<l_{2}$.

The agent 5 is invited to decrease his assessments on the alternative $x_{3}$, because $\pi\left(v_{3}^{5}, v_{3}^{+}\right)=\pi\left(l_{4}, l_{2}\right)=\delta_{4}$ and $\left(\delta_{4}, \delta_{4}\right) \prec\left(\delta_{3}, \delta_{3}\right)$.

The agents invited to increase their assessments are: 1 and 2 on the alternative $x_{2}$, because $\pi\left(v_{2}^{1}, v_{2}^{+}\right)=\pi\left(v_{2}^{2}, v_{2}^{+}\right)=\pi\left(l_{1}, l_{2}\right)=\delta_{5}$ and $\left(\delta_{5}, \delta_{5}\right) \prec$ $\left(\delta_{3}, \delta_{3}\right)$; and 1 on the alternative $x_{3}$, because $\pi\left(v_{3}^{1}, v_{3}^{+}\right)=\pi\left(l_{1}, l_{2}\right)=\delta_{5}$ and $\left(\delta_{5}, \delta_{5}\right) \prec\left(\delta_{3}, \delta_{3}\right)$.

The overall consensus reaches the fixed consensus threshold if one of the agents modifies his assessments according to the suggestions proposed by the moderator.

\section{Conclusions}

Non-uniform qualitative scales are used in different scenarios, usually through several cardinal approaches. In this paper, we have proposed a consensus reaching process in a setting where agents assess the alternatives through a non 
necessarily uniform qualitative scale, within a purely ordinal perspective. This approach is based on ordinal proximity measures that assign an abstract degree of psychological proximity to every pair of terms belonging to the qualitative scale, avoiding the widely criticized cardinal representations of linguistic terms in qualitative scales. The underlying consensus measure used in the process is of an ordinal nature: it consists of the medians of the ordinal proximities between pairs of linguistic assessments. The proposed consensus reaching process is quite flexible and it can be applied to numerous real decision problems.

It is important emphasizing that the linear order on $\Delta_{2}$ introduced in (1) and (2) is not the only one that can be considered in the sequential process, and other linear or weak orders can be used (see Bustince et al. [5], Bentkowska et al. [3] and Derrac et al. [9], among others).

We have to note that the problem of how to generate an ordinal proximity measure is not obvious. Although it has been preliminary analyzed in GarcíaLapresta and Pérez-Román [14, Subsect. 2.3], it remains an open problem.

Another open problem is to prove mathematically that the proposed consensus reaching process when implemented will ensure an increase of consensus (see $\mathrm{Wu}$ and Chiclana [24] for these kind of results in a numerical setting).

It could be interesting to devise some mechanisms for preventing strategic behaviour. One possibility is penalizing in some way those outliers that do not follow the recommendations and, consequently, difficult the increase of consensus.

\section{Acknowledgments}

The authors are grateful to Tomasa Calvo and two anonymous referees for their comments and suggestions. The financial support of the Spanish Ministerio de Economía y Competitividad (project ECO2012-32178) and Consejería de Educación de la Junta de Castilla y León (project VA066U13) are acknowledged.

\section{References}

[1] Balinski, M., Laraki, R., Majority Judgment. Measuring, Ranking, and Electing. The MIT Press, Cambridge MA, 2011.

[2] Balinski, M., Laraki, R., How best to rank wines: Majority Judgment. In: Wine Economics: Quantitative Studies and Empirical Observations, Palgrave-MacMillan, pp. 149-172, 2013.

[3] Bentkowska, U., Bustince, H., Jurio, A., Pagola, M., Pekala, B., Decision making with an interval-valued fuzzy preference relation and admissible orders. Applied Soft Computing 35, pp. 792-801, 2015.

[4] Bosch, R., Characterizations of Voting Rules and Consensus Measures. Ph. D. Dissertation, Tilburg University, 2005.

[5] Bustince, H., Fernández, J., Kolesárová, A., Mesiar, R., Generation of linear orders for intervals by means of aggregation functions. Fuzzy Sets and Systems 220, pp. 69-77, 2013. 
[6] Cabrerizo, F.J., Chiclana, F., Al-Hmouz, R., Morfeq, A., Balamash, A.S., Herrera-Viedma, E., Fuzzy decision making and consensus: challenges. Journal of Intelligent \& Fuzzy Systems 29, pp. 1109-1118, 2015.

[7] Cabrerizo, F.J., Ureña, M.R., Pedrycz, W., Herrera-Viedma, E., Building consensus in group decision making with an allocation of information granularity. Fuzzy Sets and Systems 255, pp. 115-127, 2014.

[8] Delgado, M., Vila, M.A., Voxman, W., On a canonical representation of fuzzy numbers. Fuzzy Sets and Systems 93, pp. 125-135, 1998.

[9] Derrac, J., Chiclana, F., García, S., Herrera, F., Evolutionary fuzzy knearest neighbors algorithm using interval-valued fuzzy sets. Information Sciences 329, pp. 144-163, 2016.

[10] Erdamar, B., García-Lapresta, J.L., Pérez-Román, D., Sanver, M.R., Measuring consensus in a preference-approval context. Information Fusion 17, pp. 14-21, 2014.

[11] Fedrizzi, M., Kacprzyk, J., Zadrożny, S., An interactive multi-user decision support system for consensus reaching processes using fuzzy logic with linguistic quantifiers. Decision Support Systems 4, pp. 313-327, 1988.

[12] García-Lapresta, J.L., Favoring consensus and penalizing disagreement in group decision making. Journal of Advanced Computational Intelligence and Intelligent Informatics 12 (5), pp. 416-421, 2008.

[13] García-Lapresta, J.L., Pérez-Román, D., Measuring consensus in weak orders. In: E. Herrera-Viedma, J.L. García-Lapresta, J. Kacprzyk, H. Nurmi, M. Fedrizzi, S. Zadrożny, editors. Consensual Processes, STUDFUZZ, vol. 267, Springer-Verlag, Berlin, 2011, pp. 213-234.

[14] García-Lapresta, J.L., Pérez-Román, D., Ordinal proximity measures in the context of unbalanced qualitative scales and some applications to consensus and clustering. Applied Soft Computing 35, pp. 864-872, 2015.

[15] García-Lapresta, J.L., Pérez-Román, D., Aggregating opinions in nonuniform qualitative scales. USB Proceedings of the 12th International Conference on Modeling Decisions for Artificial Intelligence (MDAI 2015), pp. 152-163, 2015.

[16] García-Lapresta, J.L., Pérez-Román, D., Consensus-based clustering under hesitant qualitative assessments. Fuzzy Sets and Systems 292, pp. 261-273, 2016.

[17] García-Lapresta, J.L., Pérez-Román, D., Falcó, E., Consensus reaching processes under hesitant linguistic assessments. In: Angelov, P. et al. (eds.), Intelligent Systems'2014, Advances in Intelligent Systems and Computing 322, pp. 257-268, 2014. 
[18] Herrera, F., Herrera-Viedma, E., Verdegay, J.L., Linguistic measures based on fuzzy coincidence for reaching consensus in group decision making. International Journal of Approximate Reasoning 16, pp. 309-334, 1997.

[19] Herrera-Viedma, E., Herrera, F., Chiclana, F., A consensus model for multiperson decision making with different preference structures. IEEE Transactions on Systems, Man and Cybernetics - Part A: Systems and Humans 32, pp. 394-402, 2002.

[20] Malkevitch, J., Mathematical theory of elections. Annals of the New York Academy of Sciences 607, pp. 89-97, 1990.

[21] Palomares, I., Estrella, F.J., Martínez, L., Herrera, F., Consensus under a fuzzy context: taxonomy, analysis framework AFRYCA and experimental case of study. Information Fusion 20, pp 252-271, 2014.

[22] Pérez, I.J., Cabrerizo, F.J., Herrera-Viedma, E., A mobile decision support system for dynamic group decision making problems. IEEE Transactions on Systems, Man and Cybernetics - Part A: Systems and Humans 40, pp. 1244-1256, 2010.

[23] Saint, S., Lawson, J.R., Rules for Reaching Consensus. A Modern Approach to Decision Making. Jossey-Bass, San Francisco, 1994.

[24] Wu, J., Chiclana, F., Multiplicative consistency of intuitionistic reciprocal preference relations and its application to missing values estimation and consensus building. Knowledge-Based Systems 71, pp. 187-200, 2014.

[25] Wu, J., Chiclana, F., Visual information feedback mechanism and attitudinal prioritisation method for group decision making with triangular fuzzy complementary preference relations. Information Sciences 279, pp. 716-734, 2014. 